\title{
Summary of session A3: mathematical studies of the field equations
}

\author{
Lars Andersson ${ }^{1}$ \\ Albert Einstein Institute, Am Mühlenberg 1, D-14476 Potsdam, Germany and Department of \\ Mathematics, University of Miami, Coral Gables, FL 33124, USA \\ E-mail: laan@aei.mpg.de
}

Received 6 November 2007

Published 15 May 2008

Online at stacks.iop.org/CQG/25/114016

\begin{abstract}
In this report I will give a summary of some of the main topics covered in session A3, mathematical studies of the field equations, at GRG18, Sydney. Unfortunately, due to length constraints, some of the topics covered at the session will be very briefly mentioned or left out altogether. The summary is mainly based on extended abstracts submitted by the speakers and some of those who presented posters at the session. I would like to thank all participants for their contributions and help with this summary.
\end{abstract}

PACS numbers: 04.20.-q, 04.25.- $\mathrm{g}, 04.40 .-\mathrm{b}$

\section{The Buchdahl inequality}

The Buchdahl inequality, which is included in most textbooks on general relativity, states that for a static, self-gravitating body,

$$
2 M / R \leqslant 8 / 9,
$$

where $M$ is the ADM mass and $R$ the area radius of the boundary of the static body. The proof by Buchdahl, cf [16] of (1) assumed that the energy density is non-increasing outwards and that the pressure is isotropic. A bound on $2 M / R$ has an immediate observational consequence: if $2 M / R<8 / 9$ then the gravitational red shift is less than 2 but if $2 M / R$ approaches 1 the red shift is unbounded. The assumptions used to derive the inequality are very restrictive, and as, e.g. pointed out by Guven and Ó Murchhada [27] neither of them hold in a simple soap bubble and they do not approximate any known topologically stable field configuration.

In addition to the restrictions implied by the hypotheses made by Buchdahl there are two other disadvantages associated with this inequality: it refers to the boundary of the body (i.e. the interior is excluded) and the solution which gives equality in the inequality is the

\footnotetext{
1 Supported in part by the NSF, under contract no DMS 0407732.
} 
Schwarzschild interior solution which has constant energy density and for which the pressure blows up at the center. In particular it violates the dominant energy condition.

Andréasson explained recent work [2], where all four restrictions described above are eliminated. He considered matter models for which the energy density $\rho$ and the radial pressure $p$ is non-negative and which satisfies $p+2 p_{T} \leqslant \Omega \rho$, where $\Omega$ is a non-negative constant and $p_{T}$ is the tangential pressure, and showed that

$$
\sup _{r>0} \frac{2 m(r)}{r} \leqslant \frac{(1+2 \Omega)^{2}-1}{(1+2 \Omega)^{2}} .
$$

Here $m$ is the quasi-local mass so that $M=m(R)$. The case $\Omega=1$ gives the bound $2 m / r \leqslant 8 / 9$. Among the matter models which satisfy the conditions stated are Vlasov matter and matter which satisfies the dominant energy condition and has non-negative pressure. Inequality (2) is sharp in the sense of measures and there are examples which come arbitrarily close to saturating the inequality. Andréasson has recently generalized the inequality to the case of charged spheres [3].

\section{Initial-boundary value problems}

A long standing problem in the analytical and numerical study of the Einstein equations is the choice of boundary conditions for boundaries near 'infinity'. As is well known, the Einstein equations in harmonic coordinates reduces to a quasilinear system of wave equations. It was proved in previous work by Kreiss and Winicour cf [36] that the initial-boundary value problem is well posed for such systems with the Sommerfeld outgoing boundary condition. The proof relied on pseudo-differential operator techniques.

Reula reported on a recent proof [35] of this fact which makes use only of partial integrations. The proof is much more transparent than the previous proof, and the argument based on partial integration is well adapted to the analysis of discretizations of the wave equation based on difference operators which have good 'partial summation' properties.

Let $\left(M, g_{a b}\right)$ be a spacetime with timelike boundary $\mathcal{T}$. Let $M$ be foliated by spacelike surfaces $\Sigma_{t}$, with timelike unit normal $n^{a}$. Consider the initial-boundary value problem for the wave equation $g^{a b} \nabla_{a} \nabla_{b} \phi=F$ with boundary condition of the form $\left.\left(T^{b}+a N^{b}\right) \nabla_{b} \phi\right|_{\mathcal{T}}=q, a>0$, where $T^{a}$ is a timelike unit vector field which is tangent to $\mathcal{T}$ and $N^{a}$ is the outward pointing normal to the boundary $\partial \Sigma_{t}=\Sigma_{t} \cap \mathcal{T}$ in $\Sigma_{t}$. The Sommerfeld condition is given by the choice $a=1$, which corresponds to a condition on the derivative of $\phi$ in an outgoing null direction. The proof proceeds by considering the fluxes of energy defined with respect to a vector field $u^{a}=T^{a}+\delta N^{a}$, where $\delta>0$ is a parameter to be chosen. Let $\Theta^{a}{ }_{b}=\nabla^{a} \phi \nabla_{b} \phi-\frac{1}{2} \delta^{a}{ }_{b} \nabla_{c} \phi \nabla^{c} \phi$ be the energy-momentum tensor for the scalar field. The energy $E$ and outward flux $\mathcal{F}$ are defined by integration over $\Sigma_{t}$ and $\partial \Sigma_{t}$, with respect to the densities $u^{b} \Theta^{a}{ }_{b} n_{a}$ and $u^{b} \Theta^{a}{ }_{b} N_{a}$, respectively. Using integration by parts one derives by a straightforward argument an energy estimate bounding $\partial_{t} E$ in terms of $E, F$ and $\mathcal{F}$. Expanding out the flux density $u^{b} \Theta^{a}{ }_{b} N_{a}$ and using the boundary condition to eliminate terms, one finds that for an appropriate choice of $\delta=\delta(a)>0$, it contains a negative multiple of the square gradient of $\phi$ on $\partial \Sigma$ plus terms which can be estimated in terms of $E, F, \mathcal{F}$. Inserting this in the above mentioned energy estimate yields a form of the energy estimate which gives the boundary regularity required for well-posedness. Based on these ideas, the proof of well-posedness for the initial boundary can now be completed along standard lines. 


\section{Moving punctures and black holes}

During the time since GRG17, Dublin 2004, there have been tremendous advances in numerical relativity, in particular in the binary black hole problem. The first breakthrough was the work by Pretorius, using a constraint stabilized harmonic coordinate formulation, with excision. Using this code he was, during the spring of 2005, able to evolve stably several orbits of a binary black hole system. This was followed shortly thereafter by announcements from several groups using different methods, that they were able to achieve similar results. One of the methods which has emerged as a successful alternative to the harmonic formulation is based on the BaumgarteShapiro-Shibata-Nakamura (BSSN) formulation of the Einstein evolution equations, cf. [7, 49], see also [48]. Due to various issues arising from a combination of several factors (gauge choice, excision etc), codes based on BSSN initially suffered from instabilities which prevented more than partial orbits to be calculated. However when the proper techniques are applied one may successfully use the BSSN formulation to stably evolve black hole spacetimes, even without excision. The black holes (BHs) are thus included in the domain of computation, and one effectively treats the BHs as essentially weak singularities in the Cauchy slices has made this approach one of the most popular in the numerical GR community. In spite of the success in using this formulation, the reason why it actually works has remained mysterious.

Husa discussed recent work, cf $[15,28]$, which sheds light on this issue. The movingpuncture method starts with BHs represented by 'filling' their interiors with wormholes, reduced to singular ('puncture') points by spatial compactification. The moving-puncture method $[6,17]$ is only a minor modification of previous approaches, in that no attempt is made to factor out the singular puncture geometry during the simulation, but surprisingly in numerical evolutions of a Schwarzschild BH using the BAM code [15], Husa et al found that this leads to a drastic change in the geometry of the time slices [28]. They have constructed an analytic solution for the stationary state of a nonspinning BH that matches the moving-puncture gauge, and found that the numerical data asymptote to this solution.

The key result about the geometry of moving punctures is that for a Schwarzschild black hole the numerical evolutions approach a stationary slice that neither reaches an internal asymptotically flat end nor hits the physical singularity, as might be expected for a stationary slice with non-negative lapse. Rather, the slice ends at a throat at finite Schwarzschild radius $\left(R_{0} \approx 1.31 M\right)$, but at infinite proper distance from the apparent horizon. This changes the singularity structure of the 'puncture'. It is still a puncture in that there is a coordinate singularity at a single point in the numerical coordinates, but it does not correspond to an asymptotically flat end. In the course of Schwarzschild evolutions Husa et al have found that the throat does collapse to the origin. Where one would have expected an inner and an outer horizon, they find only one zero in the norm of $\left(\frac{\partial}{\partial t}\right)^{a}$, corresponding to the outer horizon. An under-resolved region does develop in the spacetime (it is the region between the throat and the interior spacelike infinity), but we are pushed out of causal contact with it. The throat itself has receded to infinite proper distance from the outer horizon. Matter fields or gravitational radiation will be trapped between the inner horizon and the throat, because unlike the gauge their propagation is limited by the speed of light; Husa $\mathrm{et} \mathrm{al} \mathrm{will} \mathrm{consider} \mathrm{this} \mathrm{issue} \mathrm{in} \mathrm{future}$ work.

\section{Isolated systems}

The analysis of the asymptotic structure of asymptotically flat spacetimes is of central importance in mathematical general relativity. The conformal compactification setting 
introduced by Penrose allows one to give a stringent analysis of the asymptotic properties of spacetimes, including conserved quantities. Further, the weak cosmic censorship conjecture has a clear formulation in this setting. The work of Klainerman et al [18, 33] shows that asymptotic fall-off conditions on Cauchy data can be introduced so that the Cauchy development has a conformal compactification with any finite regularity. It is known that there are large classes of spacetimes which have a conformal compactification which is regular at spatial infinity. It is an interesting open question whether the assumption of smooth null infinity implies some type of rigidity for the spacetime [19, 21]. The regular conformal field equations of Friedrich give a clear cut formulation of this problem, and there is good hope of eventually finding sharp conditions for regularity.

\subsection{Stability of Minkowski space}

The talk by Bieri addressed the global, nonlinear stability of solutions of the Einstein equations in general relativity. In particular, she discussed results on the initial value problem for the Einstein vacuum equations, which generalizes the results of Christodoulou and Klainerman [18]. Every strongly asymptotically flat, maximal, initial data which are globally close to the trivial data gives rise to a solution which is a complete spacetime tending to the Minkowski spacetime at infinity along any geodesic. In [11], Bieri has considered the Cauchy problem with more general, asymptotically flat initial data. In particular, under the assumptions in [11], the spacetime curvature fails to be continuous. In order to show decay of the spacetime curvature and the corresponding geometrical quantities, the Einstein equations are decomposed with respect to adequate foliations of the spacetime. In the proof of Bieri, one encounters borderline estimates for certain quantities with respect to decay, indicating that the conditions concerning decay at infinity imposed on the initial data are sharp.

\subsection{Rigidity for asymptotically simple spacetimes}

Valiente Kroon reported on progress [53] toward a proof of the following conjecture concerning asymptotically simple spacetimes: if an asymptotically Euclidean, conformally flat, time symmetric initial data set for the Einstein vacuum equations gives rise to a development admitting a smooth conformal compactification at null infinity, then the initial data must be Schwarzschildean in a neighborhood of infinity. It should be noted that the Schwarzschild spacetime is the only stationary spacetime admitting conformally flat slices. There are further indications of generalizations of this conjecture for more general classes of data, cf [54, 55]. A possible proof of the above conjecture requires a detailed understanding of the structure of certain asymptotic expansions of the development of the initial data near null and spatial infinity. The tools to obtain these expansions are the 'extended conformal Einstein equations' and the representation of spatial infinity known as 'the cylinder at spatial infinity' which have been introduced by Friedrich in [25]. The asymptotic expansions are obtained by solving, for a given initial data set, a hierarchy of interior equations at the cylinder at spatial infinity. These interior equations allows us to transport initial data from the initial hypersurface up to the 'critical sets' where null infinity 'touches' spatial infinity. The structure of the interior equations suggests that their solutions should contain very specific types of logarithmic divergences at the critical sets unless the initial data is Schwarzschildean. The explicit existence of these obstructions has been shown in [53]. 


\section{Singularities}

\subsection{Stochastic aspects of generic singularities}

The proposal of Belinski, Khalatnikov and Lifshitz [9, 10, 32] (BKL) on the structure of generic cosmological singularities states roughly states that the essential properties of the asymptotic dynamics of the gravitational field along typical timelines can be understood by considering certain spatially homogeneous models. Writing the Einstein equations in terms of Hubble normalized scale invariant frame variables due to Uggla et al $[1,47,52]$ or the approach of Damour et al [22], based on Iwasawa decomposition, each of which relies on a long history of previous formulations, gives a description of the asymptotics of the gravitational field in terms of billiard dynamical systems. In the case of the Hubble normalized formulation, one gets a billiard in the Kasner plane, cf [56], while for the Iwasawa formulation one gets for the case of $3+1$ gravity, a billiard in a domain of hyperbolic space, which is analogous to the Misner-Chitre billiard.

Uggla presented work [30], building on [1, 47, 52], which generalizes and makes rigorous some aspects of the previous work of BKL and others on the stochastic aspects of the system which arise due to the chaotic nature of the asymptotic billiard systems. Using a combination of dynamical and statistical analyses and in part heuristic arguments, this work describes the generic properties of a 'billiard attractor'. It turns out that several degrees of freedom, which a priori could have been of relevance for the asymptotic behavior are, generically, statistically suppressed. The dynamical and statistical arguments of Uggla et al may be contrasted with results concerning asymptotic behavior in Bianchi type IX obtained using purely dynamical arguments [46]. The results presented by Uggla suggest that the role of Bianchi type IX as a model for the asymptotic dynamics of generic singularities should be re-evaluated, in view of the fact that generic singularities exhibit features that are not shared by Bianchi type IX.

\subsection{Kinematic and Weyl singularities}

For expanding Bianchi cosmologies with a tilted perfect fluid with linear equation of state $p=(\gamma-1) \rho$, all timelike and null geodesics are complete into the future. However, the fluid congruence may be incomplete into the future (called a congruence singularity), accompanied by the blow up of kinematic quantities associated with the fluid congruence. Lim discussed recent work [20,37] on the nature of such singularities. Much emphasis has been placed on the blow-up of the components of the Weyl tensor associated with the fluid congruence. However, as shown by examples due to Lim et al, this phenomenon is independent of the incompleteness of the fluid congruence. Hence it is necessary to differentiate congruence singularities into kinematic singularities and Weyl singularities. In particular, the fluid may become extremely titled or the kinematic or Weyl components may blow up, depending on whether $\gamma$ exceeds certain critical values.

\subsection{Perturbations of naked singularity spacetimes}

The scalar field may be considered as a toy model for perturbations of a background spacetime. Nolan discussed some rigorous mathematical results that probe the linear stability of naked singularities in self-similar collapse. He showed that the multipoles of a minimally coupled massless scalar field propagating on a spherically symmetric self-similar background spacetime admitting a naked singularity maintain finite $H^{1,2}$ norm as they impinge on the Cauchy horizon. Further, each multipole obeys a pointwise bound at the horizon, as does its locally observed energy density [40]. The null energy condition is assumed to hold in the 
background spacetime. In order to view the scalar field as a toy model for perturbations of a background spacetime, the matter model must be specified. To study such perturbations, the matter content must be specified. Nolan considers what in this context is the simplest case: that of null dust, i.e. the stability of the Cauchy horizon in self-similar Vaidya spacetime is studied. The results for the scalar field carry over to odd-parity linear perturbations at the level of both the metric and the curvature [41]. For even parity perturbations, the linearized Einstein equations can be reduced to a five-dimensional first-order symmetric hyperbolic system. The components of the state vector are gauge invariant metric and matter perturbation quantities. Nolan shows that the $L^{2}$ norm of the state vector blows up at the Cauchy horizon and that solutions for which the $L^{\infty}$ norm of the state vector does not blow-up at the Cauchy horizon are unstable in the space of test function initial data. This provides strong evidence that the Cauchy horizon of the self-similar Vaidya spacetime is unstable [39].

\subsection{Spacetime boundaries and properties of singularities}

In his talk, Whale reported on joint work with Scott, where the a-boundary and the a-boundary singularity theorem are applied to analyze the physical properties of singularities.

\section{Quasi-local aspects}

\subsection{Toward the quasi-localization of canonical GR}

Perhaps the most natural way of introducing the conserved quantities in asymptotically flat spacetimes is the canonical/Hamiltonian one. A key observation in the Hamiltonian formulation of GR, due to Arnowitt, Deser and Misner [5], is that the evolution parts of Einstein's equations can be recovered formally as canonical equations of motion in the phase space, in which the constraint function (i.e. whose vanishing is just the constraint parts of Einstein's equations) play the role of the Hamiltonian. However, as Regge and Teitelboim stressed, if we want to recover the correct evolution parts of Einstein's equations as partial differential equations for smooth fields rather than some distributional generalization of them, then the Hamiltonian in the canonical equations of motion must be functionally differentiable with respect to the canonical variables [45]. Regge and Teitelboim showed that such a Hamiltonian can be obtained by adding an appropriate 2-surface integral to the constraint functions. The remarkable property of this Hamiltonian is that the ADM energy-momentum and angular momentum can be recovered as the value of the Hamiltonian on the constraint surface with appropriately chosen lapse and shift. On the other hand, the investigations of Beig and Ó Murchadha showed that for given boundary conditions on the canonical variables the asymptotic form of the lapse and the shift is already determined if we require that the evolution equations preserve the boundary conditions [8]. Moreover, they also showed that both the constraint functions and the Hamiltonians close to Poisson algebras, the former being an ideal in the latter, and their quotient, the so-called algebra of observables, is isomorphic to the Poincare algebra. The ADM conserved quantities then can be considered as appropriate coordinates on this algebra of observables.

László Szabados considered in his talk the boundary conditions for canonical vacuum GR at the quasi-local level, i.e. when the spacelike hypersurface on which the canonical variables are defined is compact with smooth 2-boundary $\mathcal{S}$ [50]. The key ideas used by Szabados are found by analogy with the Hamiltonian analysis in the asymptotically flat case above, namely the requirement of the functional differentiability of all the functions whose Poisson bracket must be calculated, and the requirement of the compatibility of the evolution equations and the 
boundary conditions both on the canonical variables and the lapse and the shift. It has been shown by Szabados [51] that fixing the area element on the 2-surface $\mathcal{S}$ (rather than the whole induced 2-metric) is enough to have a well-defined constraint algebra, a well-defined Poisson algebra of basic Hamiltonians parametrized by lapses that are vanishing on $\mathcal{S}$ and shifts that are tangent to and divergence free on $\mathcal{S}$. Their quotient is a Lie algebra of a class of 2 -surface observables.

The evolution equations preserve these boundary conditions, and the observables (realized as the value of the basic Hamiltonians on the constraint surface) are $2+2$-covariant, gaugeinvariant and provide a representation of the Lie algebra of the divergence-free vector fields on $\mathcal{S}$. Szabados pointed out that in special, standard situations the observables appear to behave as angular momentum.

\subsection{The Einstein-scalar field at finite infinity}

The ‘finite infinity' paradigm of Ellis was proposed to study isolated gravitational systems, in our universe, where we experience the presence of other local matter fields and a cosmological background [24]. In this context it is not possible to make the assumption of asymptotic flatness at infinity, under which boundary conditions on matter have been studied in detail. Instead, a smooth timelike surface $\mathcal{F}$, is introduced. The timelike surface $\mathcal{F}$ should be located at a large finite distance from the center of the local system of interest, with the aim to study the fields generated from this on $\mathcal{F}$.

Clavering in his talk examined the behavior of the spherically symmetric Einstein-scalar field at $\mathcal{F}$. He considered the evolution of a scalar field over a domain bounded by an initial hypersurface, characteristic curves, and $\mathcal{F}$. Using Hawking's mass formula [29], he has studied the consequences of imposing conditions on the mass-energy flux at $\mathcal{F}$. Two examples were discussed; each a perturbation of a static exact solution. For a perturbation of Schwarzschild there is no mass-energy flux of the scalar field. Clavering conjectures that this is the case in all vacuum spacetimes. The analysis has been repeated for the pure scalar field case of the Wyman spacetime [57]. In this case, non-trivial expressions for the mass-energy flux in terms of the scalar field at $\mathcal{F}$ were obtained.

\subsection{Quasi-local energy inside the event horizon}

Pointlike objects cause many of the divergences that afflict physical theories. For instance, the gravitational binding energy of a point particle in Newtonian mechanics is infinite. In general relativity, the analog of a point particle is a black hole and the notion of binding energy must be replaced by quasi-local energy. The quasi-local energy (QLE) derived by York, and elaborated by Brown and York [13], is finite outside the horizon but it was not considered how to evaluate it inside the horizon.

Andrew Lundgren presented a prescription for finding the QLE inside a horizon, and showed that it is finite at the singularity for a variety of types of black holes. It turns out that the energy is typically concentrated just inside the horizon, not at the central singularity. It is impossible to localize gravitational energy due to the equivalence principle, so it is meaningless to define gravitational energy at a single point. This problem is avoided by considering the gravitational energy in a region of space. The boundary of the region is a two-dimensional surface, and in fact the quasi-local energy is defined only in terms of quantities defined on the enclosing surface (specifically the induced metric and extrinsic curvature). The QLE is defined only in terms of the gravitational degrees of freedom and makes no mention of any 
other fields that are present. However, since gravity couples to everything, the QLE counts all energy that is present in the region.

Lundgren et al [38] have considered only spherically-symmetric static black holes. The definition of the QLE in [13] was extended to be valid inside the event horizon, which is only a coordinate singularity. In the Schwarzschild case, the QLE at the central singularity is zero. The analogous quantity for a classical field of a point particle diverges at the center. The gravitational field itself carries energy which gravitates, causing the nonlinearity of general relativity. Lundgren remarked that the nonlinearity somehow provides a mechanism that cures the divergence expected of a point particle field. The definition of the QLE presented by Lundgren can be applied also to the de Sitter case and the case with non-vanishing charge.

\section{Black holes}

\subsection{Black hole rigidity and spacetimes with compact Cauchy horizon}

Isenberg reported on recent work with Moncrief which studies analytic solutions of Einstein's equations containing nondegenerate compact Cauchy horizons with non-closed generators. If certain hypotheses are added, the spacetimes necessarily admit an isometry which acts along the generators of the horizon. Isenberg showed how to use these results to prove that stationary (non-static) analytic black holes in arbitrary dimensions necessarily admit symmetries which are independent of the presumed stationarity symmetry. This work is closely related to work of Hollands et al [31].

\subsection{Electromagnetic field on the background of high dimensional black holes}

In the last few years various generalizations of black hole solutions to the high dimensional gravity have been found. One of them describes a generally rotating black hole with NUT charges. This solution possesses several nice properties as, e.g., the existence of the KillingYano tensor, the complete integrability of the geodesic motion or the separability of the Hamilton-Jacobi and Klein-Gordon equations. Its generalization including the acceleration of the black holes or the electromagnetic field is not, however, straightforward.

In four dimensions it is possible to generalize the black hole solution to describe the accelerated black holes (the Plebański-Demiański solution). This transition is achieved by the conformal rescaling of the metric accompanied by a modification of some metric functions. Krtous showed in his talk that such a procedure is not sufficiently general in higher dimensions - only the maximally symmetric spacetimes in 'accelerated' coordinates are obtained. Further, he presented general algebraically special solutions of the Maxwell equations on the background of the mentioned high dimensional generally rotating black hole. They are adjusted to the algebraic structure of the background which is reflected by the existence of the principal Killing-Yano tensor. Such electromagnetic fields generalize the field of charged black holes in four dimensions. However, one may show that in higher dimensions such fields cannot be easily modified in such a way that they would satisfy full Maxwell-Einstein equations.

\section{Post-Newtonian expansions}

Post-Newtonian expansions (PNEs) are expansions of the Einstein equations in the parameter $1 / c$, around $c=\infty$, where $c$ is lightspeed. The limit $c=\infty$ is the Newtonian limit of general relativity. Such expansions have been studied since the discovery of general relativity and 
there is a large literature available. Recently, post-Newtonian expansions have been used to calculate wave forms for the gravitational wave emissions from binary black hole inspirals. These have been compared with numerically calculated wave forms and turn out to be highly accurate until the last stage of the inspiral.

The majority of results on PNEs are based on formal expansions which are used to calculate approximate values of physical quantities, see, e.g. $[12,23]$ and references therein. However, this formal approach does not deal with the question of convergence. In the absence of a precise notion of convergence, it becomes unclear to what extent the formal expansions actually approximate relativistic solutions. In view of the importance of the applications of PNEs, it is interesting to give a rigorous foundation to the procedure.

Oliynyk discussed his recent work on post-Newtonian expansions for the Einstein-Euler equations. Oliynyk considers expansions of solutions to these equations in the parameter $\epsilon=v_{T} / c$ about $\epsilon=0$, where $v_{T}$ is a characteristic velocity scale associated with the fluid matter. By rescaling the gravitational and matter variables, the Einstein-Euler equations can be written as

$$
G^{i j}=2 \kappa \epsilon^{4} T^{i j} \quad \text { and } \quad \nabla_{i} T^{i j}=0
$$

where $\kappa=4 \pi G \rho_{T} / v_{T}^{2}, v_{i} v^{i}=-\epsilon^{-2}, \rho_{T}$ is a characteristic value for the fluid density, and $t=x^{4} / v_{T}$ is a 'Newtonian' time coordinate. In his talk, Oliynyk presented results on PNEs for a class of polytropes, which go beyond formal considerations [43, 44]. By introducing suitable renormalized variables for which the limit $\epsilon \rightarrow 0$ makes sense, and introducing a suitable gauge, Oliynyk derives a family of symmetric hyperbolic systems, depending on the parameter $\epsilon$. This system is studied in a class of weighted Sobolev spaces $H_{\delta, \epsilon}^{k}$, which interpolate between the weighted spaces $H_{\delta}^{k}$ and the standard spaces $H^{k}$. Using these spaces, it is possible to prove $\epsilon$-dependent energy estimates for solutions to the Einstein-Euler equations. These estimates are then used to prove the existence of convergent expansions in $\epsilon$ for suitably chosen initial data. Oliynyk discussed the relation between his convergent expansions and the formal PNEs. In order to recover the PNEs to a certain order requires the initial data to be chosen correctly. The method used to choose initial data is based on ideas in [14]. Oliynyk discussed how to recover the post-Newtonian expansion to second order in his framework.

\section{Miscellaneous}

\subsection{Wave tails in curved spacetimes}

Tammelo reported on joint work with Laas and Mankinen dealing with the wave tails of scalar and electromagnetic fields. Their work shows that the intensity of the tail of the electromagnetic wave pulse emitted by a wave source within a compact binary in the vicinity of the geometric shadow of the source of gravitation can be of the same magnitude as the intensity of the direct pulse. The energy carried away by the tail can amount to approximately $10 \%$ of the energy of the low-frequency modes of the direct pulse. As an example of an exactly solvable model system, a scalar wave field on a particular Robertson-Walker spacetime has been considered. In the case of minimal coupling, if the metric describes the Friedman dust-dominated universe, the higher-order multipole solutions do not contain a wave tail term. A massless nonconformally-coupled scalar field is also considered in a class of Robertson-Walker spacetimes. Tammelo et al show that an initially massless scalar field in the Robertson-Walker universe can obtain a mass in the corresponding asymptotic Minkowski space region. 


\subsection{Ricci flow and the Einstein equations}

Woolgar described work on the Ricci flow of asymptotically flat manifolds in the rotationally symmetric case [42]. This paper shows that if no minimal hypersphere is present initially, then one never forms. The flow then exists for all future time and converges to flat spacetime in the limit of infinite time, the limit being taken in the Cheeger-Gromov sense. The mass does not change during the flow, but the quasi-local mass within any fixed hypersphere about the origin (defined either by fixing the proper radius, the surface area, or the enclosed volume) evaporates away smoothly. Akbar discussed relations between Ricci solitons and solutions to the Einstein-scalar field equations.

\subsection{Posters}

Several interesting abstracts had to be relegated to the poster session. Among these were abstracts by Andréasson on the formation of black holes in spherically symmetric gravitational collapse [4], Isenberg on the AVTD behavior of $T^{2}$ symmetric solutions of the Einstein vacuum equations, Narita on cylindrically symmetric expanding spacetimes, Reula, [34], showing that the Robinson-Trautman-Maxwell equations do not constitute a well-posed initial value problem, and Valiente Kroon on a characterization of Schwarzschild initial data sets [26].

\section{References}

[1] Andersson L, van Elst H, Lim W C and Uggla C 2005 Asymptotic silence of generic cosmological singularities Phys. Rev. Lett. 94051101

[2] Andréasson $\mathrm{H} 2007$ Sharp bounds on $2 \mathrm{~m} / \mathrm{r}$ of general spherically symmetric static objects Preprint gr-qc/0702137

[3] Andréasson H 2007 Sharp bounds on the critical stability radius for relativistic charged spheres: I Preprint 0708.0219

[4] Andreasson H, Kunze M and Rein G 2007 The formation of black holes in spherically symmetric gravitational collapse Preprint 0706.3787

[5] Arnowitt R, Deser S and Misner C W 1962 The dynamics of general relativity Gravitation, an Introduction to Current Research ed L Witten (New York: Wiley) pp 227-265

[6] Baker J G, Centrella J, Choi D-I, Koppitz M and van Meter J 2006 Gravitational wave extraction from an inspiraling configuration of merging black holes Phys. Rev. Lett. 96111102

[7] Baumgarte T W and Shapiro S L 1999 On the numerical integration of Einstein's field equations Phys. Rev. D 59024007

[8] Beig R and Ó Murchadha N 1987 The Poincaré group as the symmetry group of canonical general relativity Ann. Phys., NY 174 463-98

[9] Belinski V A, Khalatnikov I M and Lifshitz E M 1970 Oscillatory approach to a singular point in the relativistic cosmology Adv. Phys. $19525-73$

[10] Belinski V A, Khalatnikov I M and Lifshitz E M 1982 A general solution of the Einstein equations with a time singularity Adv. Phys. 31 639-67

[11] Bieri L 2007 An extension of the stability theorem of the Minkowski space in general relativity PhD Thesis ETH Zurich, Switzerland

[12] Blanchet L, Faye G and Nissanke S 2005 Structure of the post-Newtonian expansion in general relativity Phys. Rev. D 72044024

[13] Brown J D and York J W Jr 1993 Quasilocal energy and conserved charges derived from the gravitational action Phys. Rev. D 47 1407-19

[14] Browning G and Kreiss H-O 1982 Problems with different time scales for nonlinear partial differential equations SIAM J. Appl. Math. 42 704-18

[15] Brügmann B et al 2008 Calibration of moving puncture simulations Phys. Rev. D 77024027

[16] Buchdahl H A 1959 General relativistic fluid spheres Phys. Rev. 116 1027-34

[17] Campanelli M, Lousto C O, Marronetti P and Zlochower Y 2006 Accurate evolutions of orbiting black-hole binaries without excision Phys. Rev. Lett. 96111101 
[18] Christodoulou D and Klainerman S 1993 The global nonlinear stability of the Minkowski space (Princeton Mathematical Series vol 41) (Princeton, NJ: Princeton University Press)

[19] Chrusciel P T and Delay E 2002 Existence of non-trivial, vacuum, asymptotically simple space-times Class. Quantum Grav. 19 L71

[20] Coley A A, Hervik S and Lim W C 2006 Fluid observers and tilting cosmology Class. Quantum Grav. 23 3573-91

[21] Corvino J and Schoen R M 2006 On the asymptotics for the vacuum Einstein constraint equations J. Differ. Geom. 73 185-217

[22] Damour T, Henneaux M and Nicolai H 2003 Cosmological billiards Class. Quantum Grav. 20 R145-200

[23] Ehlers J 1986 On limit relations between and approximative explanations of, physical theories Logic, Methodology and Philosophy of Science, VII (Salzburg, 1983) (Stud. Logic Found. Math. vol 114) (Amsterdam: North-Holland) pp 387-403

[24] Ellis G F R 2002 Cosmology and local physics New Astron. Rev. 46 645-57

[25] Friedrich H 1998 Gravitational fields near space-like and null infinity J. Geom. Phys. 24 83-163

[26] Garcia-Parrado Gomez-Lobo A and Valiente Kroon J A 2007 Initial data sets for the Schwarzschild spacetime Phys. Rev. D 75024027

[27] Guven J and Ó Murchadha N 1999 Bounds on $2 m / R$ for static spherical objects Phys. Rev. D 60084020

[28] Hannam M, Husa S, Pollney D, Bruegmann B and O’Murchadha N 2007 Geometry and regularity of moving punctures Phys. Rev. Lett. 99241102

[29] Hawking S 1968 Gravitational radiation in an expanding universe J. Math. Phys. 9 598-604

[30] Heinzle J M, Uggla C and Rohr N 2007 The cosmological billiard attractor Preprint gr-qc/0702141

[31] Hollands S, Ishibashi A and Wald R M 2007 A higher dimensional stationary rotating black hole must be axisymmetric Commun. Math. Phys. 271 699-722

[32] Khalatnikov I M, Lifshitz E M, Khanin K M, Shchur L N and Sină̌ Ya G 1984 On the stochastic properties of relativistic cosmological models near the singularity General Relativity and Gravitation (Padova, 1983) (Dordrecht: Reidel) Fund. Theories Phys. pp 343-349

[33] Klainerman S and Nicolò F 2003 Peeling properties of asymptotically flat solutions to the Enstein vacuum equations Class. Quantum Grav. $203215-57$

[34] Kozameh C, Kreiss H-O and Reula O 2008 On the well posedness of Robinson-Trautman-Maxwell solutions Class. Quantum Grav. 25025004

[35] Kreiss H O, Reula O, Sarbach O and Winicour J 2007 Well-posed initial-boundary value problem for the harmonic Einstein equations using energy estimates Class. Quantum Grav. 245973

[36] Kreiss H-O and Winicour J 2006 Problems which are well posed in a generalized sense with applications to the Einstein equations Class. Quantum Grav. 23 S405-20

[37] Lim W C, Coley A A and Hervik S 2007 Kinematic and Weyl singularities Class. Quantum Grav. 24 595-604

[38] Lundgren A P, Schmekel B S and York J W Jr 2007 Self-renormalization of the classical quasilocal energy Phys. Rev. D 75084026

[39] Nolan B C Instability of the Cauchy horizon in self-similar Vaidya space-time in preparation

[40] Nolan B C 2006 Bounds for scalar waves on self-similar naked-singularity backgrounds Class. Quantum Grav. 23 4523-37

[41] Nolan B C 2007 Odd-parity perturbations of self-similar Vaidya spacetime Class. Quantum Grav. 24 177-200

[42] Oliynyk T and Woolgar E 2007 Rotationally symmetric Ricci flow on asymptotically flat manifolds Comm. Anal. Geom. 15535

[43] Oliynyk T A 2007 The Newtonian limit for perfect fluids Comm. Math. Phys. 276131

[44] Oliynyk T A Post-Newtonian expansions for perfect fluids, in preparation

[45] Regge T and Teitelboim C 1974 Role of surface integrals in the Hamiltonian formulation of general relativity Ann. Phys., NY 88 286-318

[46] Ringström H 2001 The Bianchi IX attractor Ann. Henri Poincaré 2 405-500

[47] Röhr N and Uggla C 2005 Conformal regularization of Einstein's field equations Class. Quantum Grav. 22 3775-87

[48] Sarbach O, Calabrese G, Pullin J and Tiglio M 2002 Hyperbolicity of the BSSN system of Einstein evolution equations Phys. Rev. D 66064002

[49] Shibata M and Nakamura T 1995 Evolution of three-dimensional gravitational waves: Harmonic slicing case Phys. Rev. D 52 5428-44

[50] Szabados L B 2004 Quasi-local energy-momentum and angular momentum in GR: a review article Living Rev. Reltiv. 7 1-140 http://www.livingreviews.org/lrr-2004-4

[51] Szabados L B 2006 On a class of 2-surface observables in general relativity Class. Quantum Grav. 23 2291-302 (Preprint gr-qc/0511059) 
[52] Uggla C, van Elst H, Wainwright J and Ellis G F 2003 Past attractor in inhomogeneous cosmology Phys. Rev. D 68103502

[53] Valiente Kroon J A 2004 A new class of obstructions to the smoothness of null infinity Comm. Math. Phys. 244 133-56

[54] Valiente Kroon J A 2004 Time asymmetric spacetimes near null and spatial infinity: I. Expansions of developments of conformally flat data Class. Quantum Grav. 21 5457-92

[55] Valiente Kroon J A 2005 Time asymmetric spacetimes near null and spatial infinity: II. Expansions of developments of initial data sets with non-smooth conformal metrics Class. Quantum Grav. 22 1683-707

[56] Wainwright J and Ellis G F R 2005 Dynamical systems in cosmology Dynamical Systems in Cosmology ed J Wainwright and G F R Ellis (Cambridge: Cambridge University Press) pp 357

[57] Wyman M 1981 Static spherically symmetric scalar fields in general relativity Phys. Rev. D 24 839-41 